\title{
Serum hepcidin level and its clinical significance in maintenance hemodialysis patients
}

\author{
P. Zhang, L.-N. Yang, G. Wang, F.-E. Li, F. Tang \\ The Department of Nephrology, 452nd Hospital of the Chinese, \\ People's Liberation Army, Chengdu, China
}

Corresponding author: F. Tang

E-mail: fengtang199@126.com

Genet. Mol. Res. 13 (4): 9883-9888 (2014)

Received June 24, 2013

Accepted August 27, 2014

Published November 27, 2014

DOI http://dx.doi.org/10.4238/2014.November.27.16

\begin{abstract}
Hepcidin is a key protein of iron metabolism, which may play an important role in the prognosis of patients with chronic renal failure on maintenance hemodialysis. The purpose of this study was to investigate the relationship between the prognosis of maintenance hemodialysis patients and serum hepcidin level. We enrolled 60 patients on maintenance hemodialysis and 30 healthy controls from March 2012 to December 2012 in our hospital. Peripheral blood samples were collected to determine hepcidin by an ELISA method. Hepcidin levels of hemodialysis patients were significantly higher than those of the healthy control group. Hepcidin level was positively correlated with the degree of anemia in the dialysis group. Therefore, we conclude that hepcidin level is significantly increased in patients with chronic renal failure on maintenance hemodialysis and that increased hepcidin seriously affects the prognosis of chronic renal failure.
\end{abstract}

Key words: Hepcidin; Chronic renal failure; Maintenance hemodialysis 


\section{INTRODUCTION}

There is intracellular iron overload in end-stage renal disease (ESRD) patients, especially those on maintenance hemodialysis (MHD). This results in iron use disorder with reticuloendothelial system active due to sustained chronic inflammatory reaction (Nicolas et al., 2002a). Hepcidin is a low molecular weight peptide that was found in plasma ultrafiltrate using mass spectrometry by Krause in 2000, and it plays an important role in modulating iron homeostasis (Nicolas et al., 2002b). Intracellular iron metabolism is co-modulated by iron uptake protein (divalent metal transporter 1, DMT1) and iron export protein (ferroportin, FPN) (Nemeth et al., 2004). The main modulating sites are the duodenal enterocyte and mononuclear macrophage: FPN is located on the outer membrane of the enterocyte fundus and decreases iron uptake in the duodenum, while a decrease in DMT1 located on the cytomembrane obstructs the absorption of iron in enterocytes, resulting in a decrease in iron uptake. A decrease in macrophage FPN obstructs iron uptake in macrophages (Nemeth et al., 2004).

FPN and DMT1 on the cell surface are combined and then phosphorylated, internalized and ubiquitinated by hepcidin and finally degraded. Thus, hepcidin iron accumulation in macrophages prevents duodenal enterocytes from releasing iron into the circulation, so that there is intracellular iron overload and insufficient circulating iron (De Domenico et al., 2007; Pigeon et al., 2001). ESRD patients are always in a chronic inflammatory state. Especially for MHD patients, there is more severe inflammation with hemodialysis, which will strongly simulate the upregulation of hepcidin mRNA expression (Nicolas et al., 2002a; Vokurka et al., 2006). Meanwhile, the elimination of hepcidin decreases due to damage to renal function (Pak et al., 2006). Their co-effects weaken the downregulation of hepcidin by anemia and EPO causing a significant increase in hepcidin level in ESRD patients. This study was aimed at determining serum hepcidin level and its clinical significance in patients on MHD.

\section{MATERIAL AND METHODS}

\section{Subjects}

Sixty MHD patients seen in our hospital from March to December, 2012 and 30 healthy individuals as the control group were enrolled in this study. The MHD group of 60 cases were long-term hemodialysis patients in the Chinese People's Liberation Army 452 Hospital whose MHD term exceeded 6 months with $4 \mathrm{~h}$ per time and 2-3 times per week. The blood flow volume was $150-300 \mathrm{~mL} / \mathrm{min}$, and hemodialysis speed was $500 \mathrm{~mL} / \mathrm{min}$. Low-flux polymethylmethacrylate (PMMA) membranes and sodium bicarbonate buffer were used for all patients. Of the patients, 39 cases were male and 21 female, with an average age of $57.72 \pm$ 13.64 years old. All patients received oral ferrous succinate and intravenous r-HuEPO therapy with the administration of chalybeate and EPO for more than 4 weeks. Thirty individuals in the control group were undergoing a medical examination in the hospital during the same time, and they were healthy and without renal diseases as determined by system examination.

\section{Exclusion criteria}

The diseases that influence metabolism were excluded, such as chronic inflammation 
in recent period, other chronic infection and uncontrollable hypertension. Also excluded from the study were those on oral prophylactic, pregnant women, people receiving blood transfusion within 6 weeks, people with bleeding, and those with glutamic-pyruvic transaminase and glutamic-oxaloacetic transaminase levels 2 times more than normal (more than 45U/L), tumor, etc.

\section{Sampling}

A 2-mL non-anticoagulated blood sample was drawn from all subjects during morning before breakfast, allowed to clot at room temperature for $20 \mathrm{~min}$ and centrifuged for 10 $\mathrm{min}$ at $3000 \mathrm{rpm}$. The supernatant was carefully collected to obtain the serum, which was placed in a $-80^{\circ} \mathrm{C}$ freezer for cryopreservation. The venous blood sample was collected from MHD patients before hemodialysis.

\section{Hepcidin assay}

A competitive enzyme-linked immunosorbent assay (ELISA) was used to measure serum hepcidin. The kit is produced by American R\&B Company and uses a purified anti-human hepcidin antibody. The assay was performed strictly according to instructions.

\section{Other laboratory tests}

Iron metabolism indicator (ferritin), inflammation indicator (hsCRP), routine blood variables, etc. were tested by the clinical laboratory and biochemistry department.

\section{Statistical analysis}

The statistical software of SPSS 17.0 was adopted to conduct statistical analysis. The data are reported as means \pm standard deviation, and the statistical data were accurate to the second decimal place. The $t$-test was used for the comparison of hepcidin level between groups. The chi-square test was used for the comparison of ratios. Pearson or Spearman correlation analysis (according to whether the data showed normal distribution) was adopted for determining the correlation between hepcidin level and clinical variable. $\mathrm{P}<0.05$ was considered to be statistically significant.

\section{RESULTS}

There was no significant difference in age and gender between the 2 groups studied, but blood pressure in the MHD group was higher than that in the control. In the MHD group, the primary disease was mainly chronic glomerulonephritis which accounted for $78.33 \%$ (Table 1).

As shown in Table 2, hemoglobulin in the MHD group was significantly lower than that in the control group, while ferritin, HsCRP and hepcidin in the MHD group were significantly higher than in the control.

The correlation analyses were separately carried out between serum hepcidin level and clinical indicator in the two groups. Hepcidin level in the MHD patients and controls was positively correlated with hsCRP and ferritin, negatively correlated with hemoglobin and hematocrit, as shown in Table 3. 
Table 1. Normal clinical data comparison between the two groups.

\begin{tabular}{lccc}
\hline Characteristics & MHD group & Control group & P \\
\hline Age (year) & $57.72 \pm 13.64$ & $56.91 \pm 12.24$ & $>0.05$ \\
Gender (Male/Female) & $39 / 21$ & $18 / 12$ & $>0.05$ \\
Systolic blood pressure & $141.3 \pm 16.2$ & $72.1 \pm 10.4$ & $<0.05$ \\
Diastolic blood pressure & $94.8 \pm 14.1$ & - & $<0.05$ \\
Primary disease & $47(78.33 \%)$ & - & - \\
Chronic glomerulonephritis & $4(6.67 \%)$ & - & - \\
Hypertensive renal damage & $6(10.0 \%)$ & - & - \\
Diabetic nephropathy & $3(5.05)$ & & - \\
Polycystic kidney disease & & & \\
\hline
\end{tabular}

$\overline{\mathrm{MHD}}=$ maintenance hemodialysis.

Table 2. Test results comparison between the two groups.

\begin{tabular}{lccc}
\hline Laboratory parameters & MHD group & Control group & P \\
\hline WBC $\left(\mathrm{x} 10^{9} / \mathrm{L}\right)$ & $5.70 \pm 1.43$ & $5.91 \pm 1.39$ & $>0.05$ \\
Platelets $\left(\mathrm{x} 10^{9} / \mathrm{L}\right)$ & $144.01 \pm 43.17$ & $156.55 \pm 65.32$ & $>0.05$ \\
RBC $\left(\mathrm{x} 10^{12} / \mathrm{L}\right)$ & $3.15 \pm 0.36$ & $3.78 \pm 0.58$ & $>0.05$ \\
Hemoglobin $(\mathrm{g} / \mathrm{L})$ & $109.34 \pm 15.11$ & $125.46 \pm 17.33$ & $<0.05$ \\
hsCRP $(\mathrm{mg} / \mathrm{l})$ & $3.55 \pm 2.35$ & $1.03 \pm 0.57$ & $<0.05$ \\
Ferritin $(\mathrm{ng} / \mathrm{mi})$ & $171.12 \pm 60.54$ & $90.57 \pm 29.44$ & $<0.05$ \\
Hepcidin(ug/l) & $83.12 \pm 23.24$ & $24.12 \pm 10.21$ & $<0.05$ \\
\hline
\end{tabular}

$\mathrm{MHD}=$ maintenance hemodialysis; $\mathrm{WBC}=$ white blood cell; $\mathrm{RBC}=$ red blood cell; hsCRP $=$ high-sensitivity C-reactive protein.

Table 3. Correlation between serum hepcidin level and clinical parameters between the two groups.

\begin{tabular}{lcccr}
\hline Laboratory parameters & \multicolumn{2}{c}{ MHD group } & \multicolumn{2}{c}{ Control group } \\
\cline { 2 - 3 } \cline { 3 - 5 } & $r$ & $\mathrm{P}$ & \multicolumn{1}{c}{$r$} & 0.67 \\
\hline Ferritin & 0.79 & $<0.05$ & 0.77 & $<0.05$ \\
hSCRP & 0.81 & $<0.05$ & -0.32 & $<0.05$ \\
RBC & -0.43 & $<0.05$ & -0.29 & $<0.05$ \\
Hemoglobin & -0.24 & $<0.05$ & 0.05 \\
\hline
\end{tabular}

$\mathrm{MHD}=$ maintenance hemodialysis; hsCRP $=$ high-sensitivity $\mathrm{C}$-reactive protein; $\mathrm{RBC}=$ red blood cell.

\section{DISCUSSION}

Hepcidin was separated and purified from human blood ultrafiltrate by Krause in 2000 . Human Hepc gene is located in 19q13.1, and hepcidin is mainly synthesized and secreted by the liver (Nicolas et al., 2002b). There is rare Hepc gene expression in heart, spinal cord, lung, and renal tubular cells. The kidney is responsible for eliminating hepcidin (Pak et al., 2006), which plays an important role in modulating iron homeostasis. It makes the small intestine decrease iron uptake and reticuloendothelial system decrease iron release. Therefore, it has a negative regulatory effect on iron metabolism, and it may play a key role (Girndt et al., 1999). The expression of hepcidin is regulated by many factors. Anemia, oxygen deficiency, EPO increase, and insufficient iron downregulate hepcidin mRNA expression (Pigeon et al., 2001; Frazer et al., 2002), while inflammation, infection and iron overload upregulate its expression (Origa et al., 2007). Renal disease investigators pay more and more attention to the relationship between hepcidin and chronic renal failure complications related to iron metabolism disorder (such as 
arteriosclerosis, anemia, etc.).

In this study, a quantitative ELISA method was used to measure serum hepcidin level in MHD patients and healthy people. The hepcidin level in the MHD group was found to be significantly higher than in the control. Repeated infections in MHD patients, with blood in contact with hemodialysis membrane (Ganz, 2006), use of dialysate with endotoxin (Turgut et al., 2009) and lack of urine, may result in activation of monocytes. The activated monocyte will produce inflammation mediators such as IL-1, IL-6, IL-12 and tumor necrosis factor- $\alpha$, etc. (Horwitz and Rosenthal, 1999; Glei et al., 2002), then causing the obvious chronic inflammation in MHD patients.

The hepcidin level is negatively correlated with hemoglobin and hematocrit while positively correlated with ferritin and hsCRP in the two groups in this study. It was shown that hepcidin level is not only correlated with inflammation factors, but also significantly correlated with anemia.

There are several limitations in this study. First, it was a single center study of a small sample, so the results are not sufficient to reflect the whole MHD population. There is a need for more basic research and large-scale multiple center clinical studies to further verify the results of the present study. Second, the blood hemodialysis process may influence the hepcidin level. Because of the limited experimental conditions, the influence of the MHD process on hepcidin level was not studied. However, despite the above disadvantages in this study, it was further demonstrated that hepcidin expression level increased in MHD patients and the mechanism of anemia. The clinical meaning for determining hepcidin level in MHD patients was preliminarily discussed here.

\section{REFERENCES}

De Domenico I, Ward DM, Langelier C, Vaughn MB, et al. (2007). The molecular mechanism of hepcidin-mediated ferroportin downregulation. Mol. Biol. Cell 18: 2569-2578.

Frazer DM, Wilkins SJ, Becker EM, Vulpe CD, et al. (2002). Hepcidin expression inversely correlates with the expression of duodenal iron transporters and iron absorption in rats. Gastroenterology 123: 835-844.

Ganz T (2006). Hepcidin and its role in regulating systemic iron metabolism. Hematology Am. Soc. Hematol. Educ. Program. 29-35, 507.

Girndt M, Heisel O and Kohler H (1999). Influence of dialysis with polyamide vs haemophan haemodialysers on monokines and complement activation during a 4-month long-term study. Nephrol. Dial. Transplant. 14: 676-682.

Glei M, Latunde-Dada GO, Klinder A, Becker TW, et al. (2002). Iron-overload induces oxidative DNA damage in the human colon carcinoma cell line HT29 clone 19A. Mutat. Res. 519: 151-161.

Horwitz LD and Rosenthal EA (1999). Iron-mediated cardiovascular injury. Vasc. Med. 4: 93-99.

Krause A, Neitz S, Magert HJ, Schulz A, et al. (2000). LEAP-1, a novel highly disulfide-bonded human peptide, exhibits antimicrobial activity. FEBS Lett. 480: 147-150.

Nemeth E, Tuttle MS, Powelson J, Vaughn MB, et al. (2004). Hepcidin regulates cellular iron efflux by binding to ferroportin and inducing its internalization. Science 306: 2090-2093.

Nicolas G, Viatte L, Bennoun M, Beaumont C, et al. (2002a). Hepcidin, a new iron regulatory peptide. Blood Cells Mol. Dis. 29: 327-335.

Nicolas G, Bennoun M, Porteu A, Mativet S, et al. (2002b). Severe iron deficiency anemia in transgenic mice expressing liver hepcidin. Proc. Natl. Acad. Sci. U. S. A. 99: 4596-4601.

Nicolas G, Chauvet C, Viatte L, Danan JL, et al. (2002c). The gene encoding the iron regulatory peptide hepcidin is regulated by anemia, hypoxia, and inflammation. J. Clin. Invest. 110: 1037-1044.

Origa R, Galanello R, Ganz T, Giagu N, et al. (2007). Liver iron concentrations and urinary hepcidin in beta-thalassemia. Haematologica 92: 583-588.

Pak M, Lopez MA, Gabayan V, Ganz T, et al. (2006). Suppression of hepcidin during anemia requires erythropoietic activity. Blood 108: 3730-3735. 
Pigeon C, Ilyin G, Courselaud B, Leroyer P, et al. (2001). A new mouse liver-specific gene, encoding a protein homologous to human antimicrobial peptide hepcidin, is overexpressed during iron overload. J. Biol. Chem. 276: 7811-7819.

Turgut F, Kanbay M, Altay M, Covic A, et al. (2009). Pro-hepcidin levels in peritoneal dialysis and hemodialysis patients. Dialysis Transplantation 38: 203-209.

Vokurka M, Krijt J, Sulc K and Necas E (2006). Hepcidin mRNA levels in mouse liver respond to inhibition of erythropoiesis. Physiol. Res. 55: 667-674. 